\title{
COPPER MEASUREMENT IN EDIBLE OILS BY MODIFIED SPECTROPHOTOMETRY METHOD
}

\author{
ALI DOREHGIRAEE' ${ }^{1}$ ELHAM POURABDOLLAH ${ }^{* 2}$, MASOUD GHANEI-MOTLAGH
}

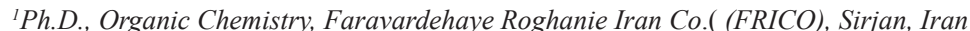 \\ ${ }^{2}$ M.S., Analytical Chemistry, Faravardehaye Roghanie Iran Co. (FRICO), Sirjan, Iran \\ ${ }^{3}$ Ph.D. student, Department of Chemistry, Faculty of Sciences, Shahid Bahonar University, Kerman, Iran.
}

\begin{abstract}
In the present study, a novel method for determination of copper content in oil samples using Neocuproine reagent was introduced. Copper ion plays a key role in environmental and biological systems which low levels of such material is necessary for living creatures. Copper is poisonous in high concentration, leaving negative effects in natural performance of human bodies. Therefore, the measuring low amount of copper is very important. Spectrophotometry methods to measure copper are based on the absorption of optically active complexes. High detection is considered as a drawback in these methods. Therefore, improving such methods by appropriate and selective complex compounds can lead to complex absorption rise and, in turn, the detection limits improvement. In the proposed method, low amounts of copper(II) ions were converted to copper(I) ions by adding the hydroxylamine hydrochloride as reducing agent. Then, the copper(I) ion was formed a yellow complex by adding 2,9dimethy 1,10 phenanthroline reagent known as Neocuproine. This method shows high sensitivity, good simplicity and accuracy, low cost, and it is applicable for determination of low copper concentration in extracted water samples from oil.
\end{abstract}

Keywords: Complex, Copper ion, Spectrophotometer, Oil, Neocuproine.

\section{INTRODUCTION}

One of industrialization side effects is using chemical materials by human beings in which their consequences can lead to various types of dangerous and fatal poisoning ${ }^{1}$. At the moment, general contamination with heavy metals is rising and, accordingly, the amounts of such materials are rising in plants and animals and consumption of their products has endangered human lives ${ }^{2}$. Food and water safety is one of major priorities in any society. Today, food supply is one of important decision-making principles for consumers to purchase food items.

Heavy metals refer to a group of metals with specific gravity of more than $5 \mathrm{~kg}$ per cubic decimeter or more than 50 atomic mass ${ }^{3}$. Presence and concentration of these elements in environment and human body leads to the development of some health problems. Some of these elements are essential for human body such as iron, copper, and zinc; however, excessive presence of such materials can be dangerous for health. The main entrance of heavy metals is via food items and water ${ }^{4}$. Ions of these metals enter the body through breathing, food, water, or skin. They will display their poisonous condition if they accumulate in the tissues of the body faster than the detoxification ${ }^{5}$.

Copper is one of essential elements in human and livestock nutrition which is effective in the production of blood hemoglobin. High concentration of copper compounds is considered as a serious threat to human health. High concentration of copper compounds can lead to development of influenza-like illness called metal fever ${ }^{6}$. Copper toxicity in children cause hyperactivity, learning disorders (such as in reading and writing disorder), and ear infections. Other symptoms of copper toxicity are autism symptoms such as depression, hallucinations, insomnia, paranoia, personality changes, dementia and schizophrenia symptoms such as increased irritability and lack of understanding of the five senses. The most common copper toxicity is damaged blood cells, lung, kidney, bad effects on liver function, and premature exhaustion. In addition, an inherited condition called Wilson's disease causes the body to retain copper and not letting it enter into the bile by the liver and eventually lead to severe brain and liver damage ${ }^{7}$. Therefore, trace analysis of copper is necessary.

Numerous instrumental technologies have been reported for the determination of copper(II). Spectrophotometry method has been popularly used for detecting of copper ions. This method is applicable in high concentration of copper; however, it is not applicable in micrograms-per-lite concentrations which is the common copper concentration in drinking water Convenience of method, inexpensive cost of material, spectrophotometer availability, and high accuracy are the main advantages of this technique. This paper aims to measure copper ion in edible oil by spectrophotometry method using hydroxylamine hydrochloride lowering and Neocuproine complex agent.

\section{Materials and methods:}

All solvents and chemical reagents used in this study were purchased from Merck (Darmstadt, Germany). The sample of crude Sunflower oil was obtained from Faravardehaye Roghanie Iran Co.(FRICO). Copper standard aqueous solution with a concentration of $1000 \mathrm{ppm}$ was purchased from Merck and it was used as a stock solution. Solutions with appropriate concentrations were prepared through diluting stock solution. Neocuproine purchased from Merck was used as copper complex reagent. Other solutions such as nitric acid, hydrogen peroxide, hydrochloric acid, ammonium acetate, and ammonium chloride hydroxide were purchased from Merck with suitable degree of decomposition. Also, ion-free water was used in all tests. A Cary 50 single detector spectrophotometer (Varian, Australia) was used for recording the absorption spectrum.

\section{Sample preparation:}

To prepare the sample, oil passes paper and then 10 gram of sample was transferred to $100 \mathrm{ml}$ Beaker and $10 \mathrm{ml}$ concentrated nitric acid $(65 \% \mathrm{~W} / \mathrm{W})$ was added. The mixture was heated for 2 hours at 90-110 Degrees Celsius for sample drying. After cooling at room temperature, $3 \mathrm{ml}$ hydrogen peroxide $(30 \% \mathrm{~V} / \mathrm{V})$ was added. In the next step, the mixture was heated until it was dried. The mixture was filtered and then it was brought to volume with $50-\mathrm{ml}$ flask with twice diluted water.

\section{Copper detection procedure:}

To ensure the removal of copper effects, all glass containers were washed with $10 \%$ nitric acid and then ion-free diluted water. In order to prepare Neocuproine reagent, 0.018 grams Neocuproine reagent was solved in $100 \mathrm{~m}$

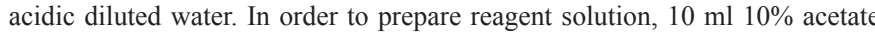
sodium solution, $5 \mathrm{ml}$ Neocuproine reagent, and $5 \mathrm{ml} 10 \%$ hydroxylamine hydrochloride solution were mixed.

\section{Standard diagram:}

In first step, copper stock solutions with proper concentration were prepared. Then, $10 \mathrm{ml}$ of each sample were transferred to tubes and $5 \mathrm{~m}$ hydroxylamine hydrochloride solution was added this solution. After that, 10 $\mathrm{ml}$ reagent solution is added to previous solution. It was mixed and left in warm water bath to change the color. Finally, sample absorption was poured in $1 \mathrm{~cm}$ cells by UV-Vis spectrophotometer at $479 \mathrm{~nm}$. For control sample, $10 \mathrm{ml}$ of diluted water was used. Test was performed for each point for three times. For oil sample, $10 \mathrm{ml}$ prepared sample was taken for measurement.

\section{Discussion:}

$\mathrm{pH}$ is one of important parameters in formation and stabilization of metal cation complexes. Also, the ability to form complex between ligand with analyte ions depends heavily on $\mathrm{pH}$ due to the presence of nitrogen atoms in 
ligand structure. Finally, the effect of solution $\mathrm{pH}$ was studied. The results are listed in Fig. 1.

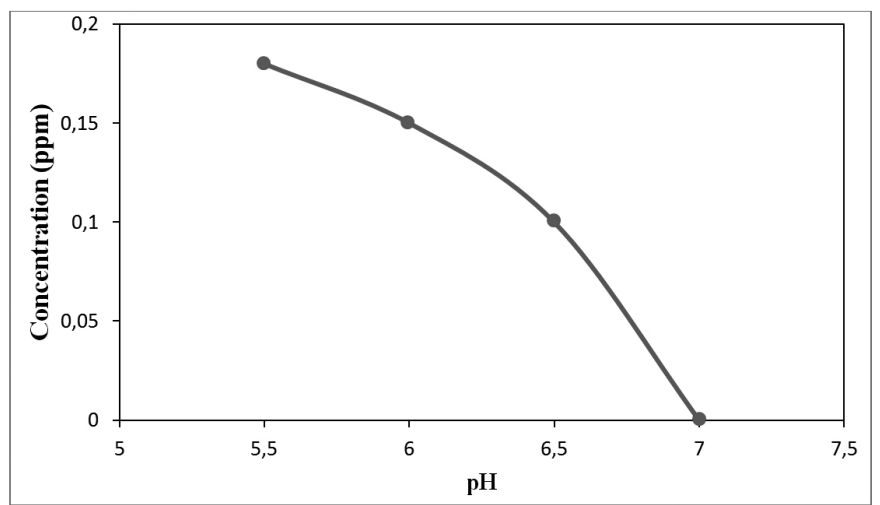

Fig. 1: The effect of solution $\mathrm{pH}$

After studying the calibration diagram in optimum condition, it is obvious that linear expansion of method is between 0.017 and $0.057 \mathrm{ppm}$. Also, the proposed method has high correlation coefficient $\left(\mathrm{R}^{2}=0.9989\right)$ between concentration and absorption in a relatively wide concentration range. Limit of detection (LOD) is $3 \mathrm{Sb} / \mathrm{m}$ and relative standard deviation is 0.017 and 0.0039 for as many as six repeated analyses, respectively.

\section{CONCLUSION}

In this paper, we introduced a simple and sensitive method to measure copper ion in edible oil samples using spectrophotometry. High correlation coefficient $\left(\mathrm{R}^{2}\right)$ between concentration and absorption in a relatively wide concentration range in measuring the analyte ion indicate the high efficiency of this method. Low detection limit in this method from one hand, and the necessity to control the amounts of heavy metals in low amounts in various food and water samples from other hand caused this method to be a strong tool to be used in different quality control labs. In addition, relatively low relative standard deviation of proposed method shows high reproducibility and proper slope in calibration curve indicates high sensitivity of this method.

\section{REFERENCES}

1. WHO, translated by NouriSepeher - Some Guidelines concerning drinking water, 2nd volume, Hayan Cultural-Publishing institute, Semnan, (1994).

2. J.E.Allen, spectrochim. Acta. 17, 467, (1961).

3. M.G.Baron, R.T.Herrin, D.E.Armstrong, Analyst, 125, 123-126, (2000).

4. M.Dabiri, Environmental pollution (air, soil, water, noise). Tehran: Alliance Publishing 58-160, (1996)

5. N.N.Greenwood, A.Earnshaw, Chemistry of the Elements. ElsevierLondon, U.K., (1997)

6. M.Resano, M.Aramendía, E.García-Ruiz, C.Crespo, M.A.Belarra, 571, 142- 149, (2006)

7. P.Shuklasr, Bioresource Technology 6, 1430-38, (2005). 